\title{
FIG4-Associated Yunis-Varon Syndrome: Identification of a Novel Missense Variant
}

\author{
Muhammad Umair ${ }^{\mathrm{a}}$ Turki M. Alkharfy $^{\mathrm{b}}$ Sajida Sajjad $^{\mathrm{c}}$ Majid Alfadhel $^{\mathrm{a}, \mathrm{d}}$ \\ a Medical Genomics Research Department, King Abdullah International Medical Research Center (KAIMRC), King Saud \\ Bin Abdulaziz University for Health Sciences, King Abdulaziz Medical City, MNGHA, Riyadh, Saudi Arabia; ${ }^{\text {Unnit Head }}$ \\ NICU, King Khalid University Hospital, Riyadh, Saudi Arabia; 'Department of Pediatrics, Family Care Hospital, Riyadh, \\ Saudi Arabia; ' ${ }^{\mathrm{G}}$ enetics and Precision Medicine Department (GPM), King Abdullah Specialized Children's Hospital \\ (KASCH), King Abdulaziz Medical City, Ministry of National Guard Health Affairs (MNG-HA), Riyadh, Saudi Arabia
}

\section{Established Facts}

- Yunis-Varon syndrome (YVS) is a severe autosomal recessive disorder characterized by skeletal defects, including cleidocranial dysplasia and digital anomalies, and severe neurological involvement with neuronal loss. Enlarged cytoplasmic vacuoles are found in neurons, muscles, and cartilage.

- YVS is caused as a result of homozygous or compound heterozygous variants in the FIG4 gene.

\section{Novel Insights}

- We characterized a Saudi proband and identified a novel homozygous missense variant (c.968A>G; p.Gln323Arg) in the FIG4 gene causing YVS.

- Our study expands the molecular and genetic spectrum of FIG4-associated mutations.

- This is the first reported case of YVS from the Saudi population.

\section{Keywords}

Yunis-Varon syndrome - Whole-exome sequencing · Cleidocranial dysplasia $\cdot$ FIG4 $\cdot$ Novel variant

\begin{abstract}
Yunis-Varon syndrome (YVS; OMIM 216340) is a rare heterogeneous autosomal recessive disorder with easy recognition of characteristic severe neurological and skeletal abnormalities involving skeletal muscles and cartilages. This cleidocranial dysplasia is characterized by bone and tooth disorders;
\end{abstract}

it also affects the cardiovascular system and tissues from ectoderm with very poor outcomes. Rarely, mutations of the FIG4 gene, encoding a 50-phosphoinositide phosphatase have been identified as the cause for YVS. We report a neonate born to a consanguineous couple with typical clinical manifestations of YVS. Using whole-exome sequencing, we identified a novel homozygous missense variant (c.968A >G; p.GIn323Arg) in the FIG4 gene. Thus, our study expands the molecular and genetic spectrum of FIG4-associated mutations. To our knowledge, this is the first reported case of YVS from the Saudi population.

○ 2021 S. Karger AG, Base
Correspondence to:

Majid Alfadhel,dralfadhelm@gmail.com 


\section{Introduction}

Yunis-Varón syndrome (YVS; OMIM 216340) is a very rare heterogeneous disorder, described for the first time in 5 infants, originated from Columbia. YVS patients exhibit clinical features such as cleidocranial dysplasia, distal aphalangia, absent halluces and thumbs, and ectodermal anomalies. Along with neurological features, the patients also present severe skeletal abnormalities. Up to date, only 26 patients with YVS from 20 families have been reported [Corona-Rivera et al., 2011; Reutter et al., 2012; Nakajima et al., 2013]. The clinical spectrum of YVS has been extended to severe limb defects with aplasia and/ or hypoplasia of nails and phalangeal bones, heart defects, earlobe dysplasia, brain malformations, retinopathy, and facial dysmorphisms [Corona-Rivera et al., 2011; Campeau et al., 2013; Nakajima et al., 2013].

Mutations in FIG4 have been associated with 4 different neurological disorders, such as autosomal recessive bilateral temporooccipital Polymicrogyria (OMIM 612691), autosomal dominant amyotrophic lateral sclerosis 11 (OMIM 612577), recessive Charcot-Marie-Tooth disease, type 4J (OMIM 611228), and recessive YVS (OMIM 216340).

Herein, we investigated a proband showing hallmark features of YVS. Whole-exome sequencing (WES) followed by Sanger sequencing identified a novel biallelic missense variant in the FIG4 gene located on chromosome 6q21.

\section{Case Presentation}

The single affected individual (II-1) in the family presented here was diagnosed having YVS on the basis of the phenotypic presentation, and molecular diagnosis. The male proband (II-1) was a product of consanguineous marriage, born at 35 weeks of gestation by cesarean section due to severe oligohydramnios to a G3P1+1 mother (Fig. 1a). The proband's (II-1) weight was $1.5 \mathrm{~kg}$, and apart from oligohydramnios, the antenatal period was normal. However, he required minimal recovery at birth and developed mild respiratory distress. The APGAR score was 6 and 8 at 1 and $5 \mathrm{~min}$, respectively.

Anthropometric data were $<3$ rd centile for gestational age. The proband had membranous skull bones with wide confluent fontanels and sutures. The proband had a high forehead, alopecia, sparse hairs, absent eyelashes/eyebrows, malformed ears, anteverted nostrils, proptosis, micrognathia, and hypertelorism (Fig. 1b, c). The pro- band had a narrow thoracic cage, which resulted in breathing difficulties and having rudimentary nipples. Features such as short limbs with absent terminal phalanges, hypoplastic thumbs, absent left big toe, and bilateral nail hypoplasia were also observed. The scrotum was empty as of undescended testis bilaterally, and there was associated hypospadias (Fig.1b, c).

The radiological examination revealed hypoplastic calvarium, bilaterally hypoplastic thin clavicles having abnormal curvature and a small thorax. Ultrasound of cranium revealed dilatation of all ventricles; these features were suggestive of mild hydrocephalus. Sonography of the abdomen revealed normal liver, spleen, and kidneys. Echocardiogram revealed biventricular hypertrophy with good left ventricular function.

The neonate was managed with oxygen support via high-flow nasal cannula for the first 2 months of life; intermittently he remained in room air only twice for about $24 \mathrm{~h}$. He had right-sided pneumothorax at 2 months of age and remained intubated for about 9 months with failed trials of extubation 8 times. At 9 months of age, a tracheostomy was carried out, and since then he is intermittently on CPAP/PS mode but mostly on synchronized intermittent mechanical ventilation support. Detail clinical description is presented in Table 1.

\section{Molecular Examination}

\section{DNA Extraction}

Blood samples were obtained from the proband and other family members. DNA extraction and quantification were performed via standard methods using commercially available genomic DNA extraction kit and a NanoDrop-1000 spectrophotometer.

\section{Whole-Exome Sequencing}

WES was performed by CENTOGENE (CentoGenome ${ }^{\circledR}$, Germany) a well-known German commercial lab. RNA capture baits against approximately $60 \mathrm{Mb}$ of human exome ( $99 \%$ target) was used to enrich the regions of interest using the fragmented DNA, using Agilent Sure-Select Human All Exon V6 kit. Using the Illumina platform the library was sequenced (coverage/ depth approx. 100×). Total, 97\% of the bases were covered at $>10 \times$. An in-house pipeline was used for base calling, alignment, and thus filtering low quality reads and artifacts. Standard annotation of the variants was applied according to the pedigree and clinical description [Asiri et al., 2020; Umair et al., 2020]. Autosomal 


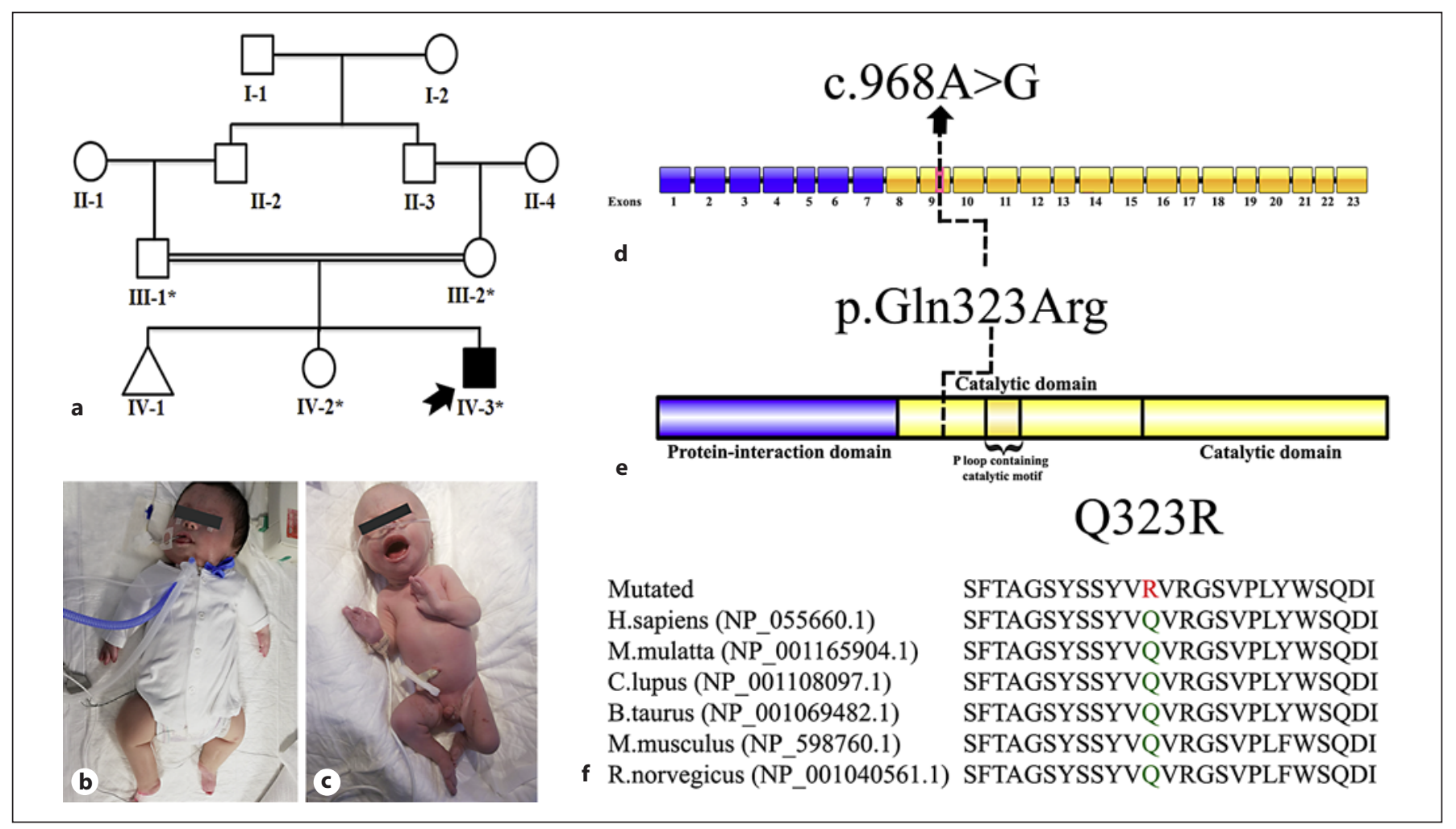

Fig. 1. a Pedigree of the family showing consanguineous union and autosomal recessive inheritance pattern. b Image of the proband (IV-3), taken immediately after birth showing typical dysmorphic facial features and limb abnormalities corresponding with YunisVaron syndrome. c Current image of the proband (IV-3) on ven-

recessive mode of inheritance was focused, homozygous, compound heterozygous variants, were given priority. All disease-causing variants with minor allele frequency of less than $1 \%$ in the gnomAD were considered.

After filtering WES data, we identified a novel homozygous missense variant (c.968A $>$ G; p.Gln323Arg) in exon 9 of the FIG4 gene (NM_014845.6), located in chromosome 6q21 (Fig. 1d). The identified variant (c.968A $>$ G) was not observed in 1000 Genomes, dbSNP, ExAC, gno$\mathrm{mAD}$, or 2000 in-house exomes.

\section{Segregation Using Sanger Sequencing}

The identified homozygous missense variant (c.968A > G; p.Gln323Arg) was Sanger sequenced in all the available family members using standard methods [Umair et al., 2016]. Primers were designed using Primer3 software (http://bioinfo.ut.ee/primer3-0.4.0/). Primer sequence can be provided upon request. tilator support and exhibiting severe developmental delay. d, e Schematic representation of the FIG4 exons and protein domains showing the position of the variant identified in the present study. f Partial sequence of FIG4, showing Gln (Q) 323 amino acid conservation across different species.

\section{In silico Analysis}

To evaluate the effect of the variants, various online protein prediction tools were used including MutationTaster, SIFT, PolyPhen-2, Varsome, Mutation assessor, and FATHMM-MKL, etc (Table 2). The variant p.(Gln323Arg) was conserved across several species (Fig. 1f).

\section{Discussion}

YVS is a severe and a rare autosomal recessive disorder characterized by severe skeletal defects, including digital anomalies, cleidocranial dysplasia, and severe neurological malformations. Affected individuals reveal enlarged cytoplasmic vacuoles in muscle, neuron, and cartilage, and the disorder is usually lethal in infancy. The condition was first reported in 5 children from 3 families with sparse hair, cleidocranial dysostosis, micrognathia, bilateral absent thumbs and first metatarsal, distal aphalangia, 
Table 1. Clinical description of the proband

\begin{tabular}{|c|c|}
\hline Clinical description & IV -3 \\
\hline Sex & Male \\
\hline Origin & Saudi \\
\hline Consanguinity & + \\
\hline Pregnancy event & Normal full term \\
\hline \multicolumn{2}{|l|}{ Head and Neck examination } \\
\hline Wide fontanelle/sutures & + \\
\hline Sparse scalp hair & + \\
\hline Protruding eyes & + \\
\hline Anteverted nostrils & + \\
\hline Dysplastic ears & + \\
\hline Microcephaly & + \\
\hline Global developmental delay & + \\
\hline Mild-intellectual disability & + \\
\hline Hypotonia & - \\
\hline Poor sleep & + \\
\hline Loose skin in neck region & + \\
\hline \multicolumn{2}{|l|}{ Limbs examination } \\
\hline Short fingers/toes & + \\
\hline Hypoplastic thumbs & + \\
\hline Hypoplastic halluces & + \\
\hline Hypoplastic nails & + \\
\hline Dysmorphic facial features & + \\
\hline MRI brain & Normal \\
\hline Skeletal survey & Abnormal \\
\hline Hearing test & Normal \\
\hline Echocardiogram & Normal \\
\hline Genetic results & $\begin{array}{l}\text { Homozygous missense } \\
\text { variant (c.968A > G; } \\
\text { p.Gln323Arg) in FIG4 }\end{array}$ \\
\hline
\end{tabular}

and bilateral hip dislocation [Yunis et al., 1980]. None of these children could survive beyond 10 weeks of age [Yunis et al., 1980]. Due to the involvement of both male and female gender and consanguinity of parents in 2 families, the authors suggested an autosomal recessive inheritance pattern. The dysmorphic features observed in our case were similar to those reported previously [Yunis et al., 1980; Campeau et al., 2013; Nakajima et al., 2013].

The features which were invariably present in all the cases reported in the literature to date include severe growth retardation, absent or hypoplastic thumbs and nails, short pointed fingers, abnormal/malformed ears, wide fontanelle with diastasis of cranial sutures, sparse scalp hairs and cleidocranial dysplasia. Other frequently reported associations were microcephaly with prominent eyes, anteverted nostrils, ectodermal abnormalities including absent or minimal eyebrows/eyelashes and hypodontia, bilateral dislocation of hips, bone fractures, and genital abnormalities including hypospadias and undescended testes. Several CNS abnormalities
Table 2. Details of the identified variant in FIG4 gene

\begin{tabular}{ll}
\hline Gene & FIG4 \\
\hline Transcript ID & NM_014845.6 \\
Chromosomal location & $6 \mathrm{q} 21$ \\
MIM number (FIG4) & 609390 \\
MIM number (YVS) & 216340 \\
Chromosomal position & 110064404 \\
Nucleotide change & c.968A>G \\
Protein change & p.Gln323Arg \\
dbSNP & rs1583671246 \\
1000G_ALL & - \\
EXAC_Freq & - \\
gnomAD & - \\
Varsome & Likely pathogenic \\
MutPred & Pathogenic \\
MutationTaster & Disease causing \\
PROVEAN & Damaging \\
SIFT & Damaging \\
REVEL & Pathogenic \\
FATHMM-MKL & Damaging \\
FATHMM-XF & Damaging \\
DANN & 0.9969-damaging \\
Mutation assessor & Medium \\
ACMG classification & Variant of uncertain \\
& significance (Class3) \\
\hline
\end{tabular}

have been reported frequently, which include DandyWalker malformation, agenesis of the corpus callosum, hydrocephalus, and hypoplastic vermis. Cardiovascular abnormalities are uncommon, but biventricular hypertrophy, cardiomyopathy and cardiomegaly have been reported with one reported case having tetralogy of Fallot. Other unique reported associations include pyloric stenosis with severe hearing impairment and papillomacular atrophic chorioretinopathy with "salt-andpepper" appearance in 2 Mexican sisters with YVS [Yunis et al., 1980; Corona-Rivera et al., 2011; Philippe et al., 2012; Campeau et al., 2013; Nakajima et al., 2013; Wright et al., 2020].

After the advent of next-generation sequencing such as whole-genome sequencing and WES, it is apparent that single mutated genes exhibiting multiple phenotypes can cause a broad phenotypic spectrum. Phenotypic overlaps have been observed in many different disorders and also in cases of YVS. In the present study, using WES, we identified a novel homozygous missense variant (c.968A $>\mathrm{G}$; p.Gln323Arg) in exon 9 of the FIG4 gene (Fig. 1d). The variant causes an amino acid change from Gln to Arg at position 323. The identified variant is present in a highly conserved catalytic domain of the FIG4 protein (Fig. 1e, f). So, far only 6 variants in the FIG5 gene have been as- 
Table 3. Mutation update reported in the FIG4 gene with autosomal recessive inheritance pattern

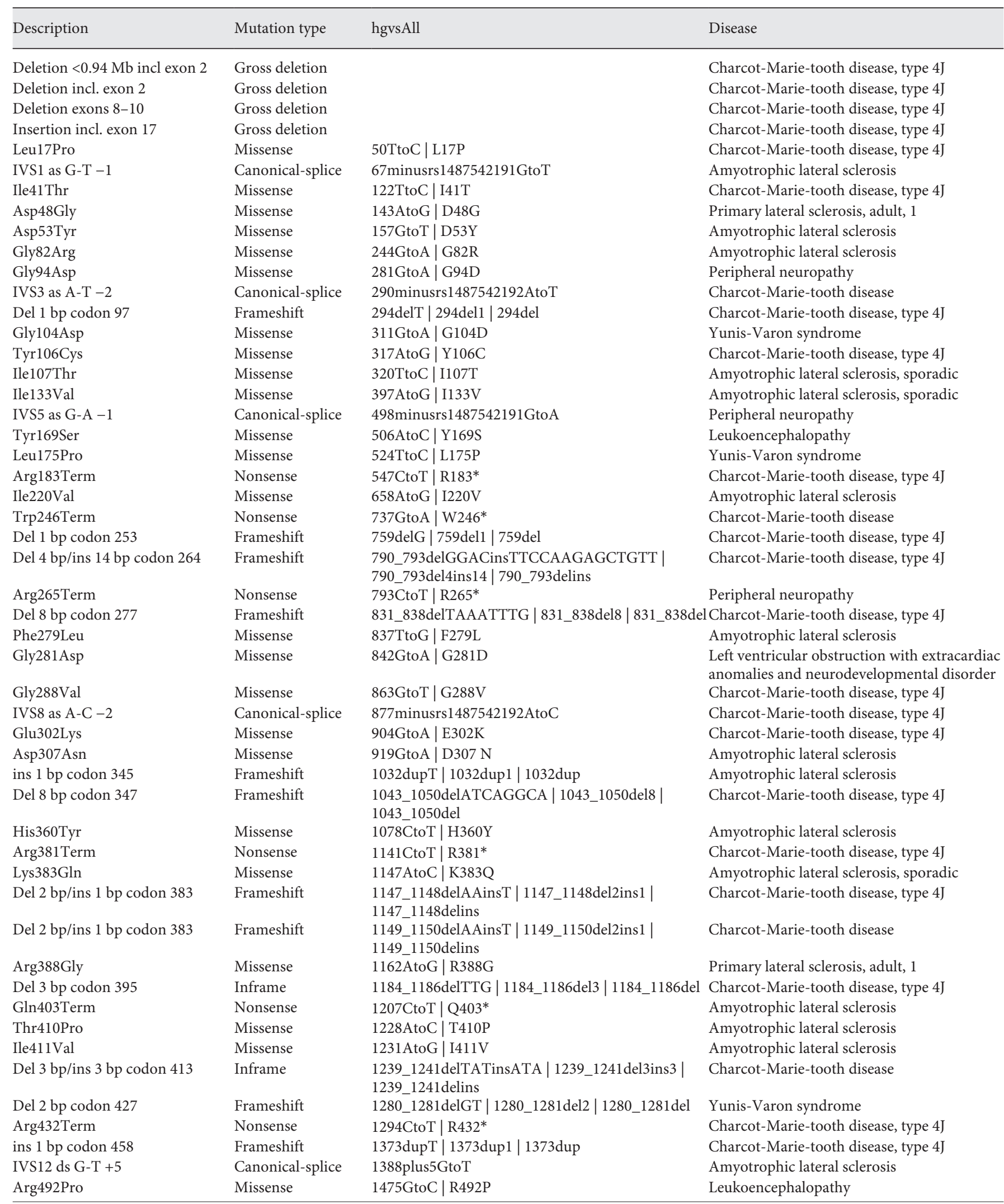


Table 3 (continued)

\begin{tabular}{|c|c|c|c|}
\hline Description & Mutation type & hgvsAll & Disease \\
\hline Del 2 bp codon 530 & Frameshift & 1589_1590delTT | 1589_1590del2 | 1589_1590del & Amyotrophic lateral sclerosis \\
\hline Thr540Ile & Missense & 1619CtoT | T540I & Amyotrophic lateral sclerosis \\
\hline ins 1 bp codon 556 & Frameshift & 1666dupA | 1666dup1 | 1666dup & Charcot-Marie-tooth disease, type 4J \\
\hline Lys559Term & Nonsense & 1675AtoT | K559* & Charcot-Marie-tooth disease, type 4J \\
\hline Del 1 bp noncoding DNA & Noncoding & $1750+1$ delG $\mid 1750+1$ del $1 \mid 1750+1$ del & Yunis-Varon syndrome \\
\hline Pro637Gln & Missense & 1910CtoA | P637Q & Amyotrophic lateral sclerosis \\
\hline Tyr647Cys & Missense & 1940AtoG | Y647C & Amyotrophic lateral sclerosis \\
\hline IVS17 as T-G -10 & Splice & 1949minusrs14875421910TtoG & Charcot-Marie-tooth disease, type 4J \\
\hline ins 1 bp codon 663 & Frameshift & 1986dupG | 1986dup1 | 1986dup & Charcot-Marie-tooth disease, type 4J \\
\hline Asp688Gly & Missense & 2063AtoG | D688G & Amyotrophic lateral sclerosis \\
\hline ins 1 bp codon 689 & Frameshift & 2065dupA | 2065dup1 | 2065dup & Peripheral neuropathy \\
\hline Met694Val & Missense & 2080AtoG | M694V & Amyotrophic lateral sclerosis \\
\hline Arg699Cys & Missense & 2095CtoT | R699C & Amyotrophic lateral sclerosis \\
\hline Arg699His & Missense & 2096GtoA | R699H & Amyotrophic lateral sclerosis \\
\hline ins 1 bp codon 718 & Frameshift & 2151dupA | 2151dup1 | 2151dup & Charcot-Marie-tooth disease, type 4J \\
\hline Del 2 bp codon 762 & Frameshift & 2285_2286delCT | 2285_2286del2 | 2285_2286del & Yunis-Varon syndrome \\
\hline ins 1 bp codon 767 & Frameshift & 2299dupG | 2299dup1 | 2299dup & Charcot-Marie-tooth disease \\
\hline Asp783Val & Missense & 2348AtoT | D783V & Epilepsy with polymicrogyria \\
\hline Gln796Term & Nonsense & 2386CtoT | Q796* & Charcot-Marie-tooth disease \\
\hline Del 3 bp codon 813 & Inframe & 2439_2441delAGA | 2439_2441del3 | 2439_2441del & Leukoencephalopathy \\
\hline Phe815Ser & Missense & 2444TtoC | F815S & Amyotrophic lateral sclerosis \\
\hline IVS21 ds G-A +1 & Canonical-splice & 2459plus1GtoA & Charcot-Marie-tooth disease \\
\hline Gln823Term & Nonsense & 2467CtoT | Q823* & Charcot-Marie-tooth disease, type 4J \\
\hline IVS22 as $\mathrm{G}-\mathrm{A}-1$ & Canonical-splice & 2547 minusrs $1487542191 \mathrm{GtoA}$ & Charcot-Marie-tooth disease \\
\hline Ser853Leu & Missense & 2558CtoT | S853L & Amyotrophic lateral sclerosis \\
\hline Arg899Term & Nonsense & 2695CtoT | R899* & Charcot-Marie-tooth disease, type 4J \\
\hline Ile902Thr & Missense & 2705TtoC | I902T & Amyotrophic lateral sclerosis \\
\hline
\end{tabular}

sociated with YVS, and this is the first case from the Saudi population (Table 3 ).

FIG4 encodes phosphatidylinositol-3,5-bisphosphatase, essential for endosome/lysosome function, and is involved in vesicle trafficking [Wright et al., 2020]. Improper function of FIG4 leads to endocytic trafficking obstruction, resulting in accumulation of vesicles in the fibroblast and peripheral neurons [Martyn and Li, 2013; Wright et al., 2020]. FIG4 binds with Fab1/PIKfyve and Vac14/ArPIKfyve, thus making a complex on the endosomal membranes known as PIKfyve-ArPIKfyve-Sac3 complex [Martyn and Li, 2013]. The complex helps in the conversion of endosomal phosphatidylinositol 3-phosphate (PI3P) to phosphatidylinositol 3,5-biphosphate (PI $(3,5) \mathrm{P} 2)$, which is essential for protein trafficking, sorting of late endosomes to lysosomal degradation compartment [Huotari and Helenius, 2011]. Mutations or improper FIG4 function results in reducing the PI $(3,5)$ $\mathrm{P} 2$, which leads to malfunctioning of the endolysosomes/ endosomes/lysosomes [Martyn and Li, 2013]. Homozygous null FIG4 mice revealed impaired growth and reduced body weight. In addition, 50\% reduction was ob- served in the volume fraction of bones, trabecular number, bone surface, and connectivity density compared to the wild type. However, the trabecular separation in the FIG4 null mice increased 3-fold. The femoral cortical thickness of the FIG4 null mice was also reduced to less than $50 \%$ compared to the normal, thus suggesting abnormal vacuolized osteoblasts and ossification [Campeau et al., 2013].

In conclusion, we revealed a novel biallelic sequence variant in the FIG4 gene associated with autosomal recessive YVS. Our finding increases the mutational spectrum of FIG4 associated with YVS. The identification of additional YVS patients will delineate the full spectrum of the disorder, expand phenotypic manifestations and the molecular mechanisms involved in the disease course.

\section{Acknowledgments}

We are grateful to the patient's family for their compliance and support. 


\section{Statement of Ethics}

All procedures were approved by the research committee of King Abdullah International Medical Research Centre (KAIMRC), Riyadh, Saudi Arabia and were performed in accordance with the Helsinki declarations. Informed consent for publication of this case report, presentation of images, and conducting research study was obtained from the parents.

\section{Conflict of Interest Statement}

The authors have no conflicts of interest to declare.

\section{Author Contributions}

M.A.: Clinical evaluation, data analysis, responsible for conception, study design, and editing the manuscript. S.S., T.M.A.: Performed clinical evaluation. M.U.: Wrote the manuscript, performed genetic evaluation, and data analysis. All the authors read and approved the final manuscript.

\section{Data Availability Statement}

The supporting data on the findings of this study can be provided by the corresponding author on reasonable request.

\section{Funding Sources}

This work was funded by the King Abdullah International Medical Research Centre.

\section{References}

Asiri A, Aloyouni E, Umair M, Alyafee Y, Al Tuwaijri A, Alhamoudi KM, et al. Mutated RAP1GDS1 causes a new syndrome of dysmorphic feature, intellectual disability \& speech delay. Ann Clin Transl Neurol. 2020 Jun;7(6): 956-64.

Campeau PM, Lenk GM, Lu JT, Bae Y, Burrage L, Turnpenny $\mathrm{P}$, et al. Yunis-Varón syndrome is caused by mutations in FIG4, encoding a phosphoinositide phosphatase. Am J Hum Genet. 2013;92(5):781-91.

Corona-Rivera JR, Romo-Huerta CO, LópezMarure E, Ramos FJ, Estrada-Padilla SA, Zepeda-Romero LC. New ocular findings in two sisters with Yunis-Varón syndrome and literature review. Eur J Med Genet. 2011; 54(1):76-81.
Huotari J, Helenius A. Endosome maturation. EMBO J. 2011;30:3481-500.

Martyn C, Li J. Fig4 deficiency: a newly emerged lysosomal storage disorder?. Prog Neurobiol. 2013;101-102:35-45.

Nakajima J, Okamoto N, Shiraishi J, Nishimura G, Nakashima M, Tsurusaki Y, et al. Novel FIG4 mutations in Yunis-Varon syndrome. J Hum Genet. 2013;58(12):822-4.

Reutter H, Bagci S, Müller A, Geipel A, Berg C, Eggermann T, et al. Primary pulmonary hypertension, congenital heart defect, central nervous system malformations, hypo and aplastic toes: another case of Yunis-Varón syndrome or report of a new entity. Eur J Med Genet. 2012;55(1):27-31.

Umair M, Hassan A, Jan A, Ahmad F, Imran M, Samman MI, et al. Homozygous sequence variants in the FKBP10 gene underlie osteogenesis imperfecta in consanguineous families. J Hum Genet. 2016;61(3):207-13.
Umair M, Ballow M, Asiri A, Alyafee Y, Al Tuwaijri A, Alhamoudi KM, et al. EMC10 homozygous variant identified in a family with global developmental delay, mild intellectual disability, and speech delay. Clin Genet. 2020; 98(6):555-61.

Wright GC, Brown R, Grayton h, Livingston JH, Park SM, Parker APJ, et al. Clinical and radiological characterization of novel FIG4-related combined system disease with neuropathy. Clin Genet. 2020;98(2):147-54.

Yunis E, Varón H. Cleidocranial dysostosis, severe micrognathism, bilateral absence of thumbs and first metatarsal bone, and distal aphalangia: a new genetic syndrome. Am J Dis Child. 1980;134(7):649-53. 